RÉNYI, A. (1958) On a one-dimensional problem concerning random space filling (in Hungarian). Magy. Tudom. Akad. Math. Kut. Intéz. Közl. 3, 109-127.

Solomon, H. (1967) Random packing density. Proc. 5th Berkeley Symp. Math. Statist. Prob. 3, 119-134.

Weiner, H. J. (1978) Sequential random packing in the plane. J. Appl. Prob. 15, 803-814.

Tokyo Institute of Technology

Yours sincerely,

MOTOO HORI

Dear Editor,

\title{
Reply to letters of
}

M. Tanemura and E. M. Tory and D. K. Pickard

I regard the arguments of [2] to be heuristic, non-rigorous and hence incomplete.

M. Tanemura's comments on Lemma 3 of [2] are well taken. In fact, it may be shown by comparison of $M(x)$ with linear solutions to the basic integral equation for $M(x)$ in the one-dimensional Rényi model ([2], p. 803, Equation (1.2)) that for $a \geqq 3 \alpha,(2.7 \mathrm{a})$ of [2] should read

$$
\left.\begin{array}{l}
(a-\alpha)^{-1} M_{\alpha}(a) \\
(b-\beta)^{-1} M_{\beta}(b)
\end{array}\right\} \text { are decreasing. }
$$

Similarly, the other ratio results of Lemma 3 of [2], p. 806 should be correspondingly changed. For example, (2.8a) should read

$$
\frac{M(a, b)}{(a-\alpha)(b-\beta)} \leqq \frac{M(c, d)}{(c-\alpha)(d-\beta)} \text { for } a \geqq c \gg \alpha, b \geqq d \gg \beta .
$$

These changes do not alter the results of [2].

The phrase ([2], p. 806, above (2.8a)), 'From the independence of $x, y$ coordinates...', refers to attempted placements, as Tory and Pickard indicate. Their examples in their Figures 1-3 and Tanemura's 'strip' example refer to particular configurations. To get the marginal density or likelihood of a 'staggered row', the conditional density for each type of configuration must be averaged with respect to its relative density of occurrence. Their comments apply only to certain configurations, not to the average over configurations. In addition, I disagree with Tanemura's remark that 'the probability of car

Received 7 May 1979. 
placement is smaller in the region where the width is narrow than in the region where it is broad and vice versa.' If the $(2 \alpha) \times(2 \beta)$ rectangles do not cut the line $l$, every space which can accommodate a car must eventually be filled, and the horizontal placement is independent of the vertical placement. The arguments of the two letters do not appear to refute the results of [2].

The computer simulations cited by Tory and Pickard all give a twodimensional limiting parking density greater than $\eta^{2}$ in the Rényi model. Their simulation yields $\operatorname{Cov}(X, Y)=0.0032$, and not 0 , as one would expect if the Palásti conjecture were true. This might be explained by the use of finite $(100 \times 100)$ parking areas for unit square cars.

The regression

$$
M(x, y)=\beta^{2}-\alpha u+o\left(\frac{1}{x}+\frac{1}{y}\right), \text { where } u=\left(\frac{1}{x}+\frac{1}{y}\right),
$$

for $u \geqq \frac{1}{50}$ may be biased in one direction due to the last term on the right of (3). As Tory and Pickard and Blaisdell and Solomon (see Tory and Pickard's letter for references) indicate, smaller values of $u$ should be most heavily weighted in the regression to determine $\beta^{2}$. Another possible effect could be that the use of periodic boundary conditions in [1] may tend to overpack the edges of the parking areas, which are the most densely packed part of the rectangles, on the average. Also, if the simulations do not exhibit that $M(x+1, y+1) / x y$ decreases as $x, y$ both increase, this can be taken as an indication that the $x \times y$ parking areas are too small to accurately exhibit the asymptotic behavior.

\section{References}

[1] JODREY, W. S. AND TORY, E. M. (1979) Random sequential packing in $\mathbb{R}^{n}$. Submitted for publication.

[2] Weiner, H. (1978) Sequential random packing in the plane. J. Appl. Prob. 15, 803-814. 